\title{
THE SPECTRUM AND FINE SPECTRUM OF GENERALIZED RHALY-CESÀRO MATRICES ON $c_{0}$ AND $c$
}

\author{
Mustafa Yildirim, MoHammad MursaleEn AND ÇAĞLA DoĞAN
}

Abstract. The generalized Rhaly Cesàro matrices $A_{\alpha}$ are the triangular matrix with nonzero entries $a_{n k}=\alpha^{n-k} /(n+1)$ with $\alpha \in[0,1]$. In [Proc. Amer. Math. Soc. 86 (1982), 405409], Rhaly determined boundedness, compactness of generalized Rhaly Cesàro matrices on $\ell_{2}$ Hilbert space and shown that its spectrum is $\sigma\left(A_{\alpha}, \ell_{2}\right)=\{1 / n\} \cup\{0\}$. Also in [32], lower bounds for these classes were obtained under certain restrictions on $\ell_{p}$ by Rhoades. In this paper, boundedness, compactness, spectra, the fine spectra and subdivisions of the spectra of generaled Rhaly Cesàro operator on $c_{0}$ and $c$ have been determined.

Mathematics subject classification (2010): Primary 40H05, 40C99, Secondary 46A35, 47A10. Keywords and phrases: Spectrum, fine spectrum, Cesàro operator, discrete generalized Cesàro operators.

\section{REFERENCES}

[1] R. Kh. Amirov, N. Durna And M. Yildirim, Subdivisions of the spectra for Cesàro, Rhaly and weighted mean operators on $c_{0}, c$ and $\ell_{p}$, IJST (2011) A3, 175-183.

[2] P. AVINOY AND P. C. TRIPATHy, The spectrum of the operator $D(r, 0,0, s)$ over the sequence spaces $\ell_{p}$ and $b v_{p}$, Hacet. J. Math. Stat. (2014) 43 (3), 425-434.

[3] A. M. AKHMEdov And F. BAŞAR, On the ne spectrum of the Cesáro operator in $c_{0}$, Math. J. Ibaraki Univ. 36 (2004), 25-32.

[4] A. M. AKHMEDov AND S. R. EL-ShABRAWy, Spectra and fine spectra of lower triangular doubleband matrices as operators on $\ell_{p}(1 \leqslant p<\infty)$, Math. Slovaca (2015) 65 (5), 1137-1152.

[5] B. AltAy AND M. KARAKUŞ, On the spectrum and the fine spectrum of the Zweier matrix as an operator on some sequence spaces, Thai J. Math. (2012) 3 (2), 153-162.

[6] M. Altun, On the fine spectra of triangular Toeplitz operators, Appl. Math. Comput (2011) 217 (20), 8044-8051.

[7] J. Appell, E. D. Pascale and A. Vignoli, Nonlinear Spectral Theory, New York, Walter de Gruyter Berlin, (2004).

[8] H. BILGIÇ AND H. FURKAN, On the fine spectrum of the generalized difference operator $B(r, s)$ over the sequence spaces $\ell_{p}$ and $b v_{p},(1 \leqslant p<\infty)$, Nonlinear Anal. 68 (3) (2008): 499-506.

[9] R. Birbonshi And P. D. SRIVAstava, On some study of the Fine Spectra of n-th band triangular matrices, Complex Anal. Oper. Theory (2016), 1-15.

[10] J. M. CARdlidge, Weighted Mean Matrices as Operators on $\ell_{p}, \mathrm{Ph}$. D. Thesis. Indiana University (1978).

[11] C. CoşKun, The spectra and fine spectra for p-Cesàro operators, Turkish J. Math. (1997) 21 (2), 207-212.

[12] R. DAS AND B. C. TRIPATHy, Spectra of the Rhaly operator on the sequence space $b v_{0} \cap \ell_{\infty}$, Bol. Soc. Paran. Mat. (2014) 3 (2), 1263-1275.

[13] R. DAS, Spectrum and fine spectrum of the Zweier matrix over the sequence space cs, Bol. Soc. Paran. Mat (2016) 35 (2), 209-221.

[14] R. DAS, On the spectrum and fine spectrum of the upper triangular matrix $U\left(r_{1}, r_{2} ; s_{1}, s_{2}\right)$ over the sequence space $c_{0}$, Afrika Mat. (2017), 1-9. 
[15] N. Durna, M. Yildirim, Subdivision of the spectra for factorable matrices on $c$ and $\ell^{p}$, Math. Commun. (2011) 16 (2), 519-530.

[16] N. DURNA, Subdivision of the spectra for the generalized upper triangular double-band matrices $\Delta^{u v}$ over the sequence spaces $c$ and $c$, ADYUSCI (2016) $6(1), 31-43$.

[17] N. Durna, M. YILDIRIM And Ü. Ç ÜnAL, On The Fine Spectrum of Generalized Lower Triangular Double Band Matrices Over The Sequence Space, Cumhuriyet Science J. (2016) 37 (3), 281-291.

[18] N. Durna, M. Yildirim And R. Kilic, Partition of the Spectra for the Generalized Difference Operator B(r,s) on the Sequence Space cs, Cumhuriyet Science J. (2018), 39 (1), 7-15.

[19] S. R. El-Shabrawy And S. H. ABU-Janah, On the Fine Structure of Spectra of Upper Triangular Double-Band Matrices as Operators on $\ell_{p}$ Spaces, Appl. Math. Inf. Sci. (2016) 10 (3), 1161-1167.

[20] S. GoldBerg, Unbounded Linear Operators, McGfraw Hill, New York, 1966.

[21] M. GonZÁLeZ, The fine spectrum of the Cesàro operator in $\ell_{p}(1<p<\infty)$, Arch. Math. (Basel) (1985) 44 (4), 355-358.

[22] J. FATHI AND R. LASHKARIPOUR, On the fine spectra of the generalized difference operator $\Delta_{u v}$ over the sequence space $c_{0}$, J. Mahani Math. Research Center (jMMRC) (2012) 1 (1) 1-12.

[23] V. KARAKAYA AND M. Altun, Fine spectra of upper triangular double-band matrices, J. Comput. Appl. Math. (2010) 234 (5), 1387-1394.

[24] V. KARAKAYA AND E. ERDOĞAN, Notes on the spectral properties of the weighted mean difference operator $G(u, v ; \Delta)$ over the sequence space $\ell_{1}$, Acta Math. Sci. (2016) 36 (2), 477-486.

[25] E. Kreyszing, Introductory Functional Analysis with Applications, John Wiley \& Sons Inc., New York Chichester Brisbane Toronto, 1978.

[26] R. LASHKARIPOUR AND J. FATHI, On the fine spectra of the Zweier matrix as an operator over the weighted sequence space $\ell_{p}(w)$, Int. J. Nonlinear Anal. Appl. (2012) 3 (1), 31-39.

[27] I. J. MAdDOX, Elements of Functional Analysis, Cambridge, University Press, (1970).

[28] J. B. ReAdE,, On the spectrum of the Cesàro operator, Bull. London Math. Soc. (1985) 17 (3), 263 267.

[29] H. C. Rhaly, JR, Discrete Generalized Cesàro Operators, Proc. Amer. Math. Soc. (1982) 86 (3), 405-409.

[30] B. E. RhoAdes, The fine spectra for weighted mean operators, Pacific J. Math. (1983), 104 (1), 219-230.

[31] B. E. RHOADES, The fine spectra for weighted mean operators in $B\left(\ell^{p}\right)$, Integral Equations Operator Theory (1989), 12 (1), 82-98.

[32] B. E. RhOADES, Lower bounds for some matrices, II, Linear And Multilinear Algebra (1990) 26 (1-2), 49-58.

[33] B. E. RhoAdes AND M. YILdirim, Spectra and fine spectra for factorable matrices, Integr. Equ. Oper. Theory (2005), 53 (1), 127-144.

[34] R. B. TAYLOR, Introduction to functional Analysis, John Wiley and Sons, 1980.

[35] B. C. TRIPAThy, A. PAUL, The spectrum of the operator $D(r, 0,0, s)$ over the sequence space $c_{0}$ and $c$, Kyungpook Math. J. (2013) $\mathbf{5 3}$ (2), 247-256.

[36] M. YEŞILKAYAGIL AND F. BAŞAR, On the fine spectrum of the operator defined by the lambda matrix over the spaces of null and convergent sequences, Abstr. Appl. Anal. 2013, Art. ID 687393, 13 pp.

[37] M. YEşILKAYAGIL AND M. KIRIŞCI, On the fine spectrum of the forward difference operator on the Hahn space, Gen. Math. Notes (2016) 33 (2), 1-16.

[38] M. YILDIRIM, The spectrum and fine spectrum of the compact Rhaly operators, Indian J. Pure Appl. Math. (1996) 27 (8), 779-784.

[39] M. YILDIRIM, The spectrum of Rhaly operators on $\ell_{p}$, Indian J. Pure Appl. Math. (2001) 32 (2), 191-198.

[40] M. YiLDiRIM, The spectrum of Rhaly operators on bvo, Indian J. Pure Appl. Math. (2003) 34 (10), $1443-1452$.

[41] Mustafa Yildirim, Nuh Durna, The spectrum and some subdivisions of the spectrum of discrete generalized Cesàro operators on $\ell^{p}(1<p<\infty)$, J. Inequal. Appl. (2017), 193, 1-13.

[42] R. B. Wenger, The fine spectra of the Hölder summability operators, Indian J. Pure Appl. Math. (1975), 6 (6), 695-712.

[43] A. Wilans Ky, Summability Through Functional Analysis, (1984) North Holland. 